\title{
¿Los Millennials Saltillenses Son Innovadores Como Consumidores de Ropa?
}

\author{
Dra. Adriana Méndez Wong \\ Mtra. Edith Reyes Ruiz \\ Dr. Jesús Francisco Mellado Siller \\ Karina Lizbeth Rodríguez, Ramírez \\ Universidad Autónoma de Coahuila, Mexico
}

Doi: 10.19044/esj.2017.v13n35p53 URL:http://dx.doi.org/10.19044/esj.2017.v13n35p53

\begin{abstract}
The objective of this research work is to analyze the behavior of young people belonging to generation $\mathrm{Y}$ known as millennials, regarding the purchase of clothing. According to Kotler and Keller (2012), a generation is a group of persons whose ages are similar and who lived in the same era. This study is considered as a descriptive and exploratory investigation. It is based on a sample of 261 persons from 21 to 36 years of age in the city of Saltillo, Coahuila. A questionnaire was applied with the scale 'Type of consumers of clothes of Chengedzai' according to the study of Manillall and Lawrence (2014). Using Pearson's chi square and an answer percentage comparison with the IBM SPSS software, the findings suggest that with regard to the innovative factor, the interviewees do not consider themselves as an innovative buyer when they buy clothes. This is because the answers concerning the variables that suggest this have a low score.
\end{abstract}

Keywords: Purchase behavior, millennials, innovative consumer

\section{Resumen}

El objetivo de este trabajo de investigación es analizar el comportamiento de los jóvenes pertenecientes a la generación $\mathrm{Y}$ conocidos como millennials, respecto a la compra de ropa. Una generación de acuerdo con Kotler \& Keller (2012) es un grupo de personas cuya edad es semejante y vivieron en una misma época. Se llevó a cabo una investigación de carácter descriptivo y exploratorio, con una muestra de 261 personas de 21 a 36 años de edad, en la ciudad de Saltillo Coahuila, aplicando un cuestionario con la escala Tipo de consumidores de ropa de Chengedzai, Manillall y Lawrence (2014) . Realizando un análisis de ji cuadrado de Pearson y un comparativo en porcentajes de las respuestas con el programa de IBM SPSS los hallazgos 
sugieren que respecto al factor Innovación, los entrevistados no se consideran comprador innovador al comprar ropa, pues evalúa bajo las respuestas a las variables que así lo sugieren.

Palabras Claves: Comportamiento de compra, millennials, consumidor innovador

\section{Introducción}

El objetivo principal de la mercadotecnia es atender a sus mercados meta, resultando imprescindible conocer los motivos de compra que subyacen a sus decisiones de compra. Cada Generación muestra rasgos y actitudes diferentes de consumo. Para la presente investigación en que se estudia el comportamiento de compra que presenta la Generación Y, con respecto a sus hábitos de compra de ropa, se advierte que los innovadores son aquellos consumidores que gustan de productos nuevos e innovadores y se emocionan buscando nuevos productos (Chengedzai, Manillall, \& Lawrence, 2014). De acuerdo con la Encuesta Intercensal 2015, en México, habitan 30.6 millones de jóvenes que representan el $25.7 \%$ de la población total (INEGI, 2017).

\section{Revisión de Literatura}

Schiffman (2010) señala que los millenials son inteligentes y prágmaticos respecto a sus hábitos de consumo; están orientados al valor; evalúan la relación precio. Calidad, es seguidor de las marcas y se opone a la publicidad exagerada.

Los innovadores son entusiastas de la tecnología a quienes les encanta probar nuevos productos y estudiar sus complejidades. A cambio de precios bajos, suelen ofrecerse como voluntarios para realizar pruebas alfa y beta, e informar a la empresa sobre las debilidades tempranas del producto. Everett Rogers define el nivel de innovación de la gente como "el grado en que una persona adopta las ideas innovadoras en un plazo relativamente menor en comparación con otros miembros de su mismo sistema social". Algunas personas son las primeras en adoptar las modas en materia de vestuario o electrodomésticos; algunos médicos son los primeros en recetar los nuevos medicamentos (Kotler \& Keller, 2012).

Los consumidores innovadores se definen como el primer 2.5 por ciento de los compradores que adoptan una nueva idea. Los innovadores son aventureros: prueban nuevas ideas con cierto riesgo. Por lo general, los innovadores tienden a ser relativamente más jóvenes, tener un nivel de educación superior y una mayor renta que los que adoptan la innovación más tarde y los que no la adoptan. Son más receptivos a las cosas con las que no están familiarizados, se fían más de sus propios valores y juicios y están más 
dispuestos a asumir riesgos, pero son menos leales a la marca y es más probable que aprovechen las promociones especiales como los descuentos, los cupones y las muestras (Kotler \& Armstrong, 2008).

Respecto al interés del tema, en el contexto científico, existen trabajos previos que analizan el comportamiento de compra de ropa:

Sproles y Kendall (1986) conceptualizan ocho características básicas de estos estilos y desarrollan un inventario de estilos de consumo para medirlas empíricamente. Llevan a cabo la validación mediante un Análisis factorial. Los resultados de la investigación se discuten y se sugieren aplicaciones del inventario de estilos de consumo en educación del consumidor, investigación del consumidor $y$ asesoramiento financiero familiar.

Para Cathy Bakewell y Vincent-Wayne Mitchell, los factores ambientales influyen de manera significativa en los compradores de la Generación Y. Realizaron un estudio en el 2003 donde examinan la toma de decisiones de los consumidores utilizando la escala Consumo Inventario de Estilos (CSI) propuesta por Sproles y de Kendall (1986). Dicho estudio utiliza la CSI como base para la segmentación de generación Y, discutiendo las implicaciones de los resultados para los minoristas y los profesionales de marketing que atienden a los consumidores de la Generación Y (Bakewell \& Mitchell, 2003).

Chengedzai, Manillall, y Lawrence (2014), realizan una investigacion donde su objetivo principal fue examinar las tipologías de compradores de la Generación Y, considerando las variables edad y moda de ropa. Utilizaron como instrumento una encuesta y su muestra fue de 230 sujetos pertenecientes a la Generación $Y$. Utilizaron el Análisis factorial exploratorio para identificar los tipos de compradores. Y aplicaron la prueba de Kruskal-Wallis para examinar la influencia de la edad sobre las tipologías de compradores identificados. El estudio identificó siete tipologías de compradores que son aplicables a los millenials: Consciente de la moda, Hedonista, Consciente de la marca, innovador, Consciente de la calidad, Indecisos, Lealtad a la marca. El valor del estudio es que estos resultados pueden permitir a los vendedores a comprender y predecir el comportamiento de compra de los consumidores de la Generación Y, facilitando el desarrollo e implementación de estrategias de marketing más eficaces.

Roehrich en 2004 (Roehrich, 2004) citado por Schiffman (Schiffman \& Lazar Kanuk, 2010) presenta una escala de medición del deseo de innovacion del consumidor con los siguientes ítems:

1. Prefiero quedarme con una marca que normalmente compro que probar algo con lo que no estoy muy seguro. 
2. Cuando voy a un restaurante, siento que es más seguro pedir platillos con los cuales estoy acostumbrado.

3. Si me gusta la marca, rara vez la cambio sólo para probar algo diferente.

4. Me gusta correr riesgos comprando marcas que no me son familiares, tan sólo para obtener algo de variedad en mis compras.

5. Cuando veo una nueva marca en el estante, no me da miedo probarla.

\section{Metodología}

Se realizó un estudio de carácter cuantitativo descriptivo y exploratorio con medición transversal no experimental, se obtuvieron 261 encuestas válidas en la ciudad de Saltillo, Coahuila. Se diseñó un instrumento de 46 ítems con escala de Likert y un análisis de las variables de Consumidor Innovador de Ropa, en la Ciudad de Saltillo, Coahuila, desde la perspectiva conceptual de la escala Tipo de consumidores de ropa de Chengedzai, Manillall y Lawrence (2014).

\section{Instrumento de Medición}

Para el presente estudio se utiliza la escala desarrollada por Chengedzai, Manillall y Lawrence (2014) y está compuesto de siete variables:

1. Consciente de la moda: Característica representativa de un consumidor quien está motivado a mantenerse al día con tendencia de moda y estilos.

2. Hedonista: Característica para medir el grado en donde un consumidor encuentra la actividad de comprar disfrutable y compra solo por diversión.

3. Consciente de la marca: Mide la orientación de un consumidor para lo más caro y de marcas reconocidas.

4. Innovador: Identifica consumidores que gustan de productos nuevos e innovadores y se emocionan buscando nuevos productos.

5. Consciente de la calidad: Característica que mide el grado en el que un consumidor busca cuidadosa y sistemáticamente la mejor calidad en productos.

6. Indecisos: Identifica al consumidor que percibe muchas marcas y tiendas de donde escoger, así como información.

7. Lealtad a la marca: Indica al consumidor que tiene marcas y tiendas favoritas y que han formado hábitos cerca de ellas.

Los Ítems del Constructo Innovador son:

1) Mantengo mi guardarropa actualizado con la moda cambiante

2) El estilo fashionista y atractivo es muy importante para mi 
3) Para tener variedad, compro en tiendas diferentes y escojo marcas diferentes

4) Es divertido comprar ropa nueva

El cuestionario se diseñó con una escala 1 a 5 tipo Likert, en donde 5 significa "totalmente de acuerdo" y 1 "totalmente en desacuerdo".

\section{Resultados}

Resultados del Estudio Empírico. Análisis descriptivo.

Perfil de los entrevistados.

Los resultados obtenidos al aplicar el instrumento a un grupo de 261 personas de la Generación "Y" en la ciudad de Saltillo Coahuila, respecto a los factores sociodemográficos, presentan resultados preliminares siendo los más relevantes los siguientes: Los millennials sus edades oscilan entre $21 \mathrm{y}$ 35 años; el 51.7\% de los entrevistados son hombres y el 48.3\% son mujeres; el $66.7 \%$ cuenta con estudios de Licenciatura y el $18.4 \%$ bachillerato; en relación con el estado civil el 67.8\% manifiesta ser soltero.

Se lleva a cabo un análisis comparativo en porcentajes con el programa IBM SPSS, del Constructo Innovador, y al preguntarles "Mantengo mi guardarropa actualizado con la moda cambiante" los resultados fue la respuesta Totalmente en desacuerdo con un $32.6 \%$ la que obtuvo mayor porcentaje pues representó para los hombres con un $18.8 \% \mathrm{y}$ $13.8 \%$ para las mujeres; respecto a la pregunta "El estilo fashionista y atractivo es muy importante para mi " la opción totalmente en desacuerdo es la respuesta más recurrente con un $38.3 \%$ siendo $22.6 \%$ para los hombres y un $15.7 \%$ para las mujeres. Al cuestionarles "Para tener variedad, compro en tiendas diferentes y escojo marcas diferentes" los valores más altos se obtienen en la respuesta valor tres "Indeciso" con un $31.4 \%$ siendo los hombres con un $16.1 \%$ y las mujeres con un $15.3 \%$. Respecto a si les parece divertido comprar ropa nueva, la opción Indeciso es la de mayor porcentaje en su respuesta con un $33.7 \%$ con un $18.8 \%$ para el género masculino y un 14.9\% para el género femenino.

Dimensión Innovador: Tabulación cruzada "Mantengo mi ropa actualizado con la moda cambiante" y Género.

Análisis Ji cuadrado de Pearson

Hipótesis

H0: Mantengo mi ropa actualizada es independiente del género.

H1: Hay relación entre el género y mantengo mi ropa actualiza.

$\mathrm{H} 1$ : No existen diferencias significativas entre hombres y mujeres con respecto a mantener la ropa actualizada con la moda cambiante. 
Tabla 1. Ji cuadrado de Pearson

\begin{tabular}{|c|c|c|c|c|c|c|}
\hline & \multicolumn{4}{|c|}{ Mantengo mi guardarropa actualizado } & \multirow{2}{*}{ Total } \\
\cline { 2 - 6 } & $\begin{array}{c}\text { Totalmente } \\
\text { Desacuerdo }\end{array}$ & Desacuerdo & Indeciso & Acuerdo & $\begin{array}{c}\text { Totalmente De } \\
\text { Acuerdo }\end{array}$ & \\
\hline \multirow{2}{*}{ Genero } & & & & 18 & 1 & 135 \\
HOMBRE & 49 & 32 & 35 & 18 & 11 & 126 \\
MUJER & 36 & 26 & 42 & 11 & 12 & 261 \\
\hline
\end{tabular}

Pruebas de chi-cuadrado

\begin{tabular}{|c|c|c|c|}
\hline & Valor & gl & Sig. asintótica (bilateral) \\
\hline Chi-cuadrado de Pearson & $12.973^{\mathrm{a}}$ & 4 & .011 \\
Razón de verosimilitudes & 14.403 & 4 & .006 \\
Asociación lineal por lineal & 4.234 & 1 & .040 \\
N de casos válidos & 261 & & \\
\hline
\end{tabular}

a. 0 casillas $(0.0 \%)$ tienen una frecuencia esperada inferior a 5. La frecuencia mínima esperada es 5.79.

En las Tablas 1, y $1^{\text {a }}$ se encuentran los resultados de la ji cuadrado de Pearson que muestra una significancia > a.05, no se rechaza la H0, por lo que se puede afirmar con una confianza al $95 \%$ que no hay diferencias significativas entre hombres y mujeres con respecto a mantener la ropa actualizada con la moda cambiante.

Tabla 2. Tabla de contingencia Género y Mantengo mi ropa actualizada

\begin{tabular}{|c|c|c|c|c|c|c|}
\hline & \multicolumn{5}{|c|}{ Mantengo mi guardarropa actualizado } & \multirow[t]{2}{*}{ Total } \\
\hline & $\begin{array}{l}\text { Totalmente } \\
\text { Desacuerdo }\end{array}$ & Desacuerdo & Indeciso & Acuerdo & $\begin{array}{c}\text { Totalmente De } \\
\text { Acuerdo }\end{array}$ & \\
\hline \multirow[t]{2}{*}{ HOMBRE } & 49 & 32 & 35 & 18 & 1 & 135 \\
\hline & $18.8 \%$ & $12.3 \%$ & $13.4 \%$ & $6.9 \%$ & $0.4 \%$ & $51.7 \%$ \\
\hline \multirow[t]{2}{*}{ MUJER } & 36 & 26 & 42 & 11 & 11 & 126 \\
\hline & $13.8 \%$ & $10.0 \%$ & $16.1 \%$ & $4.2 \%$ & $4.2 \%$ & $48.3 \%$ \\
\hline TOTAL & $32.6 \%$ & $22.2 \%$ & $29.5 \%$ & $11.1 \%$ & $4.6 \%$ & $100.0 \%$ \\
\hline
\end{tabular}

En la Tabla 2 se encontró que la respuesta Totalmente en desacuerdo contiene los valores más altos con un $32.6 \%$, mientras que Totalmente de acuerdo es la respuesta con más bajo porcentaje $4.6 \%$, en la respuesta Indeciso se obtiene un $29.5 \%$, en Desacuerdo un $22.2 \%$ y de Acuerdo solo un $11.1 \%$.

Tabla 3. Tabla de contingencia Género y el estilo fashionista y atractivo es muy importante para mi

\begin{tabular}{|c|c|c|c|c|c|c|}
\hline & \multicolumn{5}{|c|}{ El estilo fashionista y atractivo es muy importante para mi } & \multirow[t]{2}{*}{ Total } \\
\hline & $\begin{array}{l}\text { Totalmente } \\
\text { Desacuerdo }\end{array}$ & Desacuerdo & Indeciso & Acuerdo & $\begin{array}{c}\text { Totalmente De } \\
\text { Acuerdo }\end{array}$ & \\
\hline HOMBRE & 59 & 30 & 25 & 16 & 5 & 135 \\
\hline & $22.6 \%$ & $11.5 \%$ & $9.6 \%$ & $6.1 \%$ & $1.9 \%$ & $51.7 \%$ \\
\hline MUJER & 41 & 23 & 36 & 19 & 7 & 126 \\
\hline & $15.7 \%$ & $8.8 \%$ & $13.8 \%$ & $7.3 \%$ & $2.7 \%$ & $48.3 \%$ \\
\hline TOTAL & $38.3 \%$ & $20.3 \%$ & $23.4 \%$ & $13.4 \%$ & $4.6 \%$ & $100.0 \%$ \\
\hline
\end{tabular}


En la Tabla 3 al preguntarles si el estilo fashionista es muy importante, los entrevistados consideran estar totalmente en desacuerdo con un $38.3 \%$, Indeciso es la respuesta en segundo sitio con un $23.4 \%$, en Desacuerdo con un $20.3 \%$. Un $13.4 \%$ está de acuerdo con la afirmación y un 4.6\% si está totalmente de acuerdo.

Tabla 4. Tabla de contingencia Género y para tener variedad compro en tiendas diferentes

\begin{tabular}{|c|c|c|c|c|c|c|}
\hline & \multicolumn{4}{|c|}{ Para tener variedad, compro en tiendas diferentes } & \multirow{2}{*}{ Total } \\
\cline { 2 - 6 } & $\begin{array}{c}\text { Totalmente } \\
\text { Desacuerdo }\end{array}$ & Desacuerdo & Indeciso & Acuerdo & $\begin{array}{c}\text { Totalmente De } \\
\text { Acuerdo }\end{array}$ & \\
\hline HOMBRE & 32 & 28 & 42 & 16 & 17 & 135 \\
& $12.3 \%$ & $10.7 \%$ & $16.1 \%$ & $6.1 \%$ & $6.5 \%$ & $51.7 \%$ \\
MUJER & 26 & 24 & 40 & 17 & 19 & 126 \\
& $10.0 \%$ & $9.2 \%$ & $15.3 \%$ & $6.5 \%$ & $7.3 \%$ & $48.3 \%$ \\
TOTAL & $22.2 \%$ & $19.9 \%$ & $31.4 \%$ & $12.6 \%$ & $13.8 \%$ & $100.0 \%$ \\
\hline
\end{tabular}

La Tabla 4 nos muestra que los entrevistados en un $31.4 \%$ se encuentran indecisos de comprar en tiendas diferentes para obtener variedad en su guardarropa; un $22.2 \%$ si están totalmente en desacuerdo con la afirmación; un 19.9\% está en desacuerdo en comparación con un $13.8 \%$ que está totalmente de acuerdo. Y por último un $12.6 \%$ está de acuerdo en buscar variedad de ropa en tiendas diferentes.

Tabla 5. Tabla de contingencia Género y es divertido comprar ropa nueva

\begin{tabular}{|c|c|c|c|c|c|c|}
\hline & \multicolumn{5}{|c|}{ Es divertido comprar ropa nueva } & \multirow[t]{2}{*}{ Total } \\
\hline & $\begin{array}{l}\text { Totalmente } \\
\text { Desacuerdo }\end{array}$ & Desacuerdo & Indeciso & Acuerdo & $\begin{array}{l}\text { Totalmente De } \\
\text { Acuerdo }\end{array}$ & \\
\hline HOMBRE & $\begin{array}{c}30 \\
11.5 \%\end{array}$ & $\begin{array}{c}29 \\
11.1 \%\end{array}$ & $\begin{array}{c}49 \\
18.8 \%\end{array}$ & $\begin{array}{c}11 \\
4.2 \%\end{array}$ & $\begin{array}{c}16 \\
6.1 \%\end{array}$ & $\begin{array}{c}135 \\
517 \%\end{array}$ \\
\hline MUJER & 20 & 23 & 39 & 10 & 34 & 126 \\
\hline TOTAL & $\begin{array}{c}7.7 \% \\
19.2 \%\end{array}$ & $\begin{array}{c}8.8 \% \\
19.9 \%\end{array}$ & $\begin{array}{l}14.9 \% \\
33.7 \%\end{array}$ & $\begin{array}{l}3.8 \% \\
8.0 \%\end{array}$ & $\begin{array}{l}13.0 \% \\
19.2 \%\end{array}$ & $\begin{array}{c}48.3 \% \\
100.0 \%\end{array}$ \\
\hline
\end{tabular}

En la Tabla 5 se observa que un $33.7 \%$ es indeciso al responder que si encuentra diversión por comprar ropa nueva; con el mismo porcentaje de $19.2 \%$ están totalmente de acuerdo y en total desacuerdo con la afirmación; un $19.9 \%$ manifiesta desacuerdo y un $8 \%$ está de acuerdo en divertirse comprando ropa nueva.

\section{Discusión}

En las primeras líneas de este trabajo se justifica el interés por el estudio del comportamiento de compra específicamente de ropa. Hay que añadir, además la escasez de trabajos empíricos existentes en México, lo que destaca la pertinencia de estudios como éste.

En concreto, el estadístico Ji cuadrado de Pearson nos señala que no 
se rechaza la $\mathrm{H} 0$, pues se muestra que no hay diferencias significativas entre hombres y mujeres con respecto a mantener la ropa actualizada con la moda cambiante.

El estudio presenta algunas limitaciones, la muestra para el análisis estadístico es pequeña, por lo que no se pueden generalizar los resultados. Un siguiente estudio deberá incluir una muestra más grande, contemplando sujetos de otras generaciones, para poder así, comparar los grupos. Pero en general se percibe que los consumidores de ropa en Saltillo tienden a ser tradicionales en su forma de vestir.

\section{Conclusión}

El marketing generacional pretende atender con estrategias más efectivas que sean incluyentes y al mismo tiempo, ofrecer algo a cada segmento. Los primeros hallazgos al realizar un análisis de pertenencia de género y las variables de consumidor de moda Innovador, es que no existen diferencias significativas en los hombres y mujeres al mantener el guardarropa actualizado coincidiendo con el estudio de Chengedzai, Manillall y Lawrence (2014), entre los consumidores millennials, que no hace diferencias con la variable género, y generaliza sus resultados entre ambos sexos.

Los resultados nos muestran que en general, la población entrevistada no se considera comprador innovador al compar ropa, pues evalúa bajo las respuestas a las variables que así lo sugieren.

\section{References:}

1. Schiffman, L. G., \& Lazar Kanuk, L. (2010). Comportamiento del Consumidor (DÉCIMA EDICIÓN ed.). México: PEARSON EDUCACIÓN.

2. Bakewell, C., \& Mitchell, V.-W. (2003). "Generation Y female consumer decision-making styles". International Journal of Retail \& Distribution Management, 31(2), 95-106.

3. Borrás Catalá, V. (2007). Inequalities in consumption through. Revista Española de Sociología, 139-156.

4. Carosio, A. (2008). Revista de estudios de género. La ventana. Recuperado el 2017, de El género del consumo en la sociedad de consumo.:

http://www.scielo.org.mx/scielo.php?script=sci_arttext\&pid=S140594362008000100006\&lng=es\&tlng=es.

5. Chengedzai , M., Manillall , D., \& Lawrence, M. (2 de Abril de 2014). 'Shopper typologies amongst a Generation Y consumercohort and variations in terms of age in the fashionapparel market'. Recuperado el 26 de Enero de 2015, de 
http://www.actacommercii.co.za:

http://dx.doi.org/10.4102/ac.v14i1.209

6. Fan, J. X., \& Xiao, J. J. (1998). Consumer decision-making styles of young-adult Chinese. . Journal of Consumer Affairs, , 275-294.

7. Hair, J. F., E., A. R., Tatham, R. L., \& Black, W. C. (1999). Análisis Multivariante (quinta Edición). Madrid, España.: Pearson Educación.

8. Hernández, S. R., Fernández, C. C., \& Baptista, L. P. (2014). Metodología de la Investigación (Sexta Edición). México: McGrawHill.

9. INEGI. (2017). "ESTADÍSTICAS A PROPÓSITO DEL DIA INTERNACIONAL DE LA JUVENTUD. Aguascalientes: INEGI.

10. Kotler, P. A. (2007). Marketing. Versión para Latinoamérica. Mexico: Pearson.

11. Kotler, P., \& Armstrong, G. (2008). Fundamentos de Marketing. México: Pearson Prentice Hall $8^{\circ}$ Edición.

12. Kotler, P., \& Keller, k. (2012). Dirección de Marketing. México: Pearson Educación.

13. Moreno, O. J. (2005). Analisis Multivariante en Investigaciones de Calidad del Servicio. Revista Venezolana de Analisis de Coyontura, 275-308.

14. Muñoz Molina, Y. (2004). La satisfacción del consumidor en las experiencias hedonistas. Academia. Revista Latinoamericana de Administración, 52-67.

15. Nunnally, J. C. (1987). Teoría Psicométrica. México: Ed. Trillas .

16. O'Shaughenessy, J., \& O'Shaughenenessy, N. (2002). "Marketing, the Consumer Society and Hedonism". European Journal of Marketing, 36, 524-548.

17. Roehrich, G. (2004). Consumer Innovativeness: Concepts and measurements. Journal of Business Research, 674.

18. Sprotles, G. B., \& Kendall, E. L. (1986). A Methodology for Profiling Consumers' Decision-Making Styles. Journal of Consumer Affairs, 267-279. 